\title{
The effect of longitudinal flow on resonantly damped kink oscillations
}

\author{
J. Terradas ${ }^{1}$, M. Goossens ${ }^{1}$, and I. Ballai ${ }^{2}$ \\ 1 Centre Plasma Astrophysics and Leuven Mathematical Modeling and Computational Science Center, \\ Katholieke Universiteit Leuven, Celestijnenlaan 200B, 3001 Leuven, Belgium \\ e-mail: [jaume; marcel.goossens] @wis . kuleuven. be \\ 2 Solar Physics and Space Plasmas Research Centre $\left(\mathrm{SP}^{2} \mathrm{RC}\right)$, Department of Applied Mathematics, University of Sheffield, \\ Hounsfield Road, Hicks Building, Sheffield, S3 7RH, England, UK \\ e-mail: I.Ballai@sheffield.ac.uk
}

Received 16 October 2009 / Accepted 1 March 2010

ABSTRACT

\begin{abstract}
Context. The most promising mechanism acting towards damping the kink oscillations of coronal loops is resonant absorption. In this context most of previous studies neglected the effect of the obvious equilibrium flow along magnetic field lines. The flows are in general sub-Alfvénic and hence comparatively slow.

Aims. Here we investigate the effect of an equilibrium flow on the resonant absorption of linear kink MHD waves in a cylindrical magnetic flux tube with the aim of determining the changes in the frequency of the forward and backward propagating waves and in the modification of the damping times due to the flow.

Methods. A loop model with both the density and the longitudinal flow changing in the radial direction is considered. We use the thin tube thin boundary (TTTB) approximation in order to calculate the damping rates. The full resistive eigenvalue problem is also solved without assuming the TTTB approximation.

Results. Using the low ratio of flow and Alfvén speeds we derive simple analytical expressions to the damping rate. The analytical expressions are in good agreement with the resistive eigenmode calculations.

Conclusions. Under typical coronal conditions the effect of the flow on the damped kink oscillations is weak when the characteristic scale of the density layer is similar or lower than the characteristic width of the velocity layer. However, in the opposite situation the damping rates can be significantly altered, specially for the backward propagating wave which is undamped while the forward wave is overdamped.
\end{abstract}

Key words. magnetohydrodynamics (MHD) - waves - magnetic fields - Sun: corona

\section{Introduction}

In space and solar plasmas flows are observed in high resolution on almost all temporal and spatial scales. Flows are ubiquitous in active region loops and the measurements of their velocities have been provided by instruments like SoHO (see Brekke et al. 1997; Winebarger et al. 2002), TRACE (see Winebarger et al. 2001) and more recently Hinode (see for example Chae et al. 2008; Ofman \& Wang 2008; Terradas et al. 2008b). In general the flow speeds are low, and in most of the observations they are subAlfvénic, typically less that $10 \%$ of the Alfvén speed. The bulk motions are observed along magnetic field lines which outline coronal loops. These flows could be generated by some catastrophic cooling of coronal loops or are related to some siphon mechanism arising due to the difference in pressure at the loop footpoints. Since longitudinal steady flows carrying momentum and providing additional inertia are present in coronal loops it is necessary to study their effects on the transverse oscillations observed in these structures (see for example Aschwanden et al. 1999; Nakariakov et al. 1999). An effect which is of obvious importance for magnetohydrodynamic (MHD) wave theory of loops' dynamics is how the period and the damping time are modified by the flow.

Here we are interested in the damping of the fundamental kink mode due to resonant absorption, based on the transfer of energy from a global MHD wave to local resonant Alfvén waves, and in the way the efficiency of the mechanism is altered by a stationary flow. In the past, the influence of a velocity shear on this process due to a longitudinal flow has been studied by Hollweg et al. (1990); Peredo \& Tataronis (1990), Ruderman \& Goossens (1995); Erdélyi et al. (1995); Erdélyi \& Goossens (1996); Tirry et al. (1998). More recently, Andries et al. (2000); Andries \& Goossens (2001); Erdélyi \& Taroyan (2003a,b), have investigated in detail resonant flow instabilities which can occur for velocity shears significantly below the Kelvin-Helmholtz (KH) threshold. These instabilities are produced when the frequency of the forward propagating wave (propagating in the direction of the flow) shifts into the Doppler shifted continuum of the backward propagating wave. Under these conditions the mode becomes unstable and the flow acts at the resonant layer as an energy source. In most of the aforementioned studies it has been assumed that the wavelength is shorter or similar to the tube radius. This is not the case for standing kink oscillations in coronal loops which are precisely in the opposite regime, i.e., where the thin tube (TT) approximation is applicable.

In the present paper we extend the previous studies about resonant absorption in the presence of flow to the situation where the TT approximation is valid. We start by reviewing the properties of kink MHD waves in a homogeneous tube with an axial flow and study the nature of the waves, that 
change from being trapped to leaky and eventually become $\mathrm{KH}-$ unstable. Then we consider a non-uniform tube whose oscillation is damped by resonant absorption and investigate how the damping time is modified by the flow. In the following analysis three different approaches are implemented. Firstly, we use existing theoretical work, mainly by Goossens et al. (1992), to calculate the changes in the period and damping rates induced by the longitudinal flow using the thin tube thin boundary approximation (TTTB). Secondly, under the TTTB assumption we derive a linear analytical approximation to the damping rate and thirdly, solve the full resistive eigenvalue problem without the TTTB assumption. Reassuringly, we find that the three methods lead to essentially the same results.

\section{Basic features: uniform tube}

We consider what we can call the standard loop model, a cylindrical axi-symmetric flux tube of radius $R$ with a constant axial magnetic field $B_{0}$ and with a density contrast of $\rho_{\mathrm{i}} / \rho_{\mathrm{e}}$ where the indices " $i$ " and " $e$ " describe quantities inside and outside the loop, respectively. Inside the loop there is an axial flow denoted by $v_{\mathrm{i}}$. For simplicity we assume that there is no flow outside the tube so that $v_{\mathrm{e}}=0$. We start by recalling the analytical results obtained for a uniform loop (no transition layer) in the $\beta=0$ case. It is well-known that the effect of the flow introduces a shift in the frequency of waves and that the known expressions for the dispersion relation without flow can be used by simply replacing the frequency $\omega$ by its Doppler-shifted counterpart, $\Omega=\omega-k v$, $k$ being the wavenumber along the tube. The dispersion relation of MHD waves was derived in Goossens et al. (1992) (see also Terra-Homem et al. 2003; Soler et al. 2008). In the TT approximation $(k R \ll 1)$ Goossens et al. (1992) found that the frequency of the kink MHD wave modified by the flow, $\omega_{\mathrm{kf}}$, (see their equation Eq. [83]) is given by

$\omega_{\mathrm{kf}}=k \frac{\rho_{\mathrm{i}} v_{\mathrm{i}}}{\rho_{\mathrm{i}}+\rho_{\mathrm{e}}} \pm \omega_{\mathrm{cm}}$,

where $\omega_{\mathrm{kf}}$ is the frequency of the kink wave modified by the flow, and $\omega_{\mathrm{cm}}$ is the frequency in the centre-of-mass frame as in Hollweg et al. (1990),

$\omega_{\mathrm{cm}}=\left\{\omega_{\mathrm{k}}^{2}-\frac{\rho_{\mathrm{i}} \rho_{\mathrm{e}}}{\left(\rho_{\mathrm{i}}+\rho_{\mathrm{e}}\right)^{2}} k^{2} v_{\mathrm{i}}^{2}\right\}^{1 / 2}$.

In the above equation $\omega_{\mathrm{k}}$ is the classic kink frequency of a thin static tube and is given by

$\omega_{\mathrm{k}}=\left\{\frac{\rho_{\mathrm{i}} \omega_{\mathrm{A}, \mathrm{i}}^{2}+\rho_{\mathrm{e}} \omega_{\mathrm{A}, \mathrm{e}}^{2}}{\rho_{\mathrm{i}}+\rho_{\mathrm{e}}}\right\}^{1 / 2}$,

with $\omega_{\mathrm{A}}$ being the local Alfvén frequency, defined as

$\omega_{\mathrm{A}}=k v_{\mathrm{A}}$.

The frequency $\omega_{\mathrm{kf}}$ of the MHD waves is degenerate with respect to the azimuthal wave number in the TT approximation. Our interest is in the kink waves with azimuthal number $m=1$ since these waves are the ones that move the axis of symmetry and the loop as a whole. The plus and minus sign in front of the second term of Eq. (1) represent two different waves, one propagating in the same direction of the flow (forward) and the other propagating against the flow (backward). The presence of the flow breaks the degeneracy of positive and negative frequencies present when $v_{\mathrm{i}}=v_{\mathrm{e}}=0$.
The second term of Eq. (1) contains the condition for the Kelvin-Helmholtz instability which occurs for any velocity shear in absence of a magnetic field. When magnetic fields are present it is straightforward to see that the square root in Eq. (1) is negative when the flow is faster than the critical value given by

$\frac{v_{\mathrm{i}}}{v_{\mathrm{Ai}}} \geq \sqrt{2\left(1+\frac{\rho_{\mathrm{i}}}{\rho_{\mathrm{e}}}\right)}$.

The above condition means that fast flows compared to the internal Alfvén speed are required for the Kelvin-Helmholtz instability to occur (see also Chandrasekhar 1961; Ferrari et al. 1981). An equivalent problem has been studied in the context of propagating transverse waves in coronal jets by Vasheghani Farahani et al. (2009). These authors have also found that in the observationally determined range of parameters, the waves do not undergo either to the KHI or the negative energy wave instability.

However, before the Kelvin-Helmholtz instability occurs, it may happen that the frequency of the modes is above the external cut-off frequency $\left(\omega_{\mathrm{A}, \mathrm{e}}=k v_{\mathrm{A}, \mathrm{e}}\right)$, meaning that the wave becomes leaky. The forward propagating wave becomes leaky when the following condition is satisfied

$\frac{v_{\mathrm{i}}}{v_{\mathrm{A}, \mathrm{i}}} \geq \sqrt{\frac{\rho_{\mathrm{i}}}{\rho_{\mathrm{e}}}}-1$.

Similar to the KH-instability, fast flows are required to generate a leaky wave. Contrary to the static situation studied by Goossens et al. (2009), in the presence of flow an underdense loop is not required to have leaky modes when $\beta=0$. Solving the dispersion relation in the TT approximation, the damping of waves due to leakage is derived from the imaginary part of the frequency given by

$\gamma=-\frac{\pi}{4}\left(k_{\mathrm{e}} R\right)^{2} \frac{\rho_{\mathrm{e}}\left(\omega_{\mathrm{kf}+}^{2}-\omega_{\mathrm{A}, \mathrm{e}}^{2}\right)}{\left(\rho_{\mathrm{i}}+\rho_{\mathrm{e}}\right) \omega_{\mathrm{cm}}}$.

The decrement is proportional to the square of $k R\left(k_{\mathrm{e}} \sim k\right)$, meaning that leakage is basically unimportant in the TT approximation since the damping time $\left(\tau_{\mathrm{D}}=1 / \gamma\right)$ is very large. Thus, the damping due to MHD radiation in the presence of flow is a very inefficient damping mechanism for the transverse loop oscillations, even in the presence of fast flows.

An example of the dependence of the real $\left(\omega_{\mathrm{R}}\right)$ and imaginary $(\gamma)$ part of the frequency of the modes (fundamental forward and backward waves) on the flow is shown in Fig. 1 for a particular equilibrium configuration $\left(L=100 R, \rho_{\mathrm{i}} / \rho_{\mathrm{e}}=3\right)$. The domains where waves become leaky or when a KHI occurs are clearly shown. When the frequencies of the two modes merge for increasing velocity shear $\left(v_{\mathrm{i}}\right)$ the system becomes unstable. However, note that the forward wave is always leaky before the system becomes KH unstable (compare also Eq. (6) with Eq. (5)). It is important to mention here that we have not considered here the Principal Leaky Mode which is a very peculiar solution of the dispersion relation (see Cally 1986, 2003) and instead have focused on the modes than are trapped for a the static background.

Once we know the main effects of the flow on the kink MHD waves we need to know in which regime of Fig. 1 we can match, for example, the observed standing kink oscillations. The observations of flows in coronal loops indicate that they are slow, therefore hereafter we focus on sub-Alfvénic flows rather than the super-Alfvénic flows that might cause leakage and $\mathrm{KH}$ instabilities. We concentrate on the regime $v_{\mathrm{i}} / v_{\mathrm{Ai}}<0.1$, thus according to the previous analysis both the forward and backward 


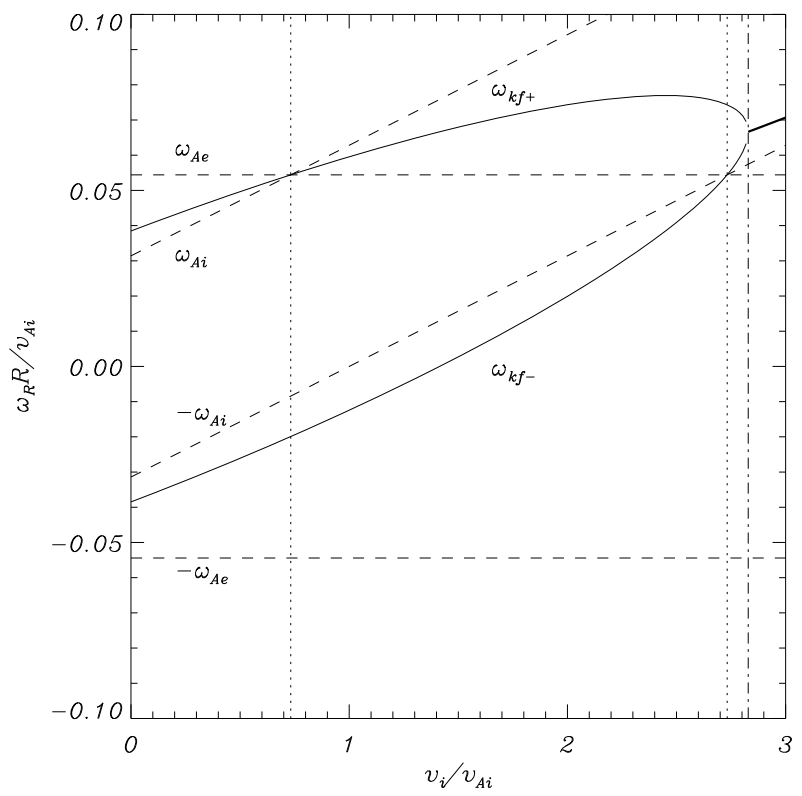

(a)

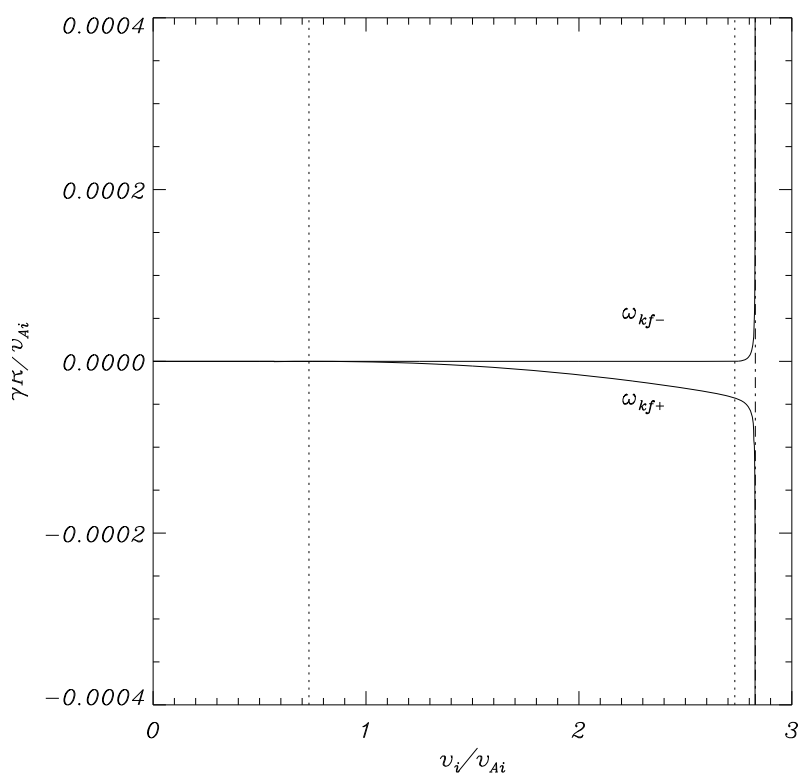

(b)

Fig. 1. a) Real and b) imaginary part of the frequency of the forward $\left(\omega_{\mathrm{kf}+}\right)$ and backward $\left(\omega_{\mathrm{kf}-}\right)$ propagating waves as a function of the internal flow $\left(v_{\mathrm{i}}\right)$ in units of the internal Alfvén speed. The curves intersect at the KH critical flow, see dot-dashed line, given by Eq. (5). The forward wave becomes leaky before the KHI criteria, since the frequency of the mode crosses the external Alfvén frequency $\left(\omega_{\mathrm{Ae}}\right)$, see dotted lines, at the value of the flow given by Eq. (6) (in the TT approximation). The dashed lines represent the Doppler shifted Alfvén frequencies. In this plot $L=100 R, \rho_{\mathrm{i}} / \rho_{\mathrm{e}}=3$.

waves are always trapped. This also prevents the presence of resonant flow instabilities which occur when the frequency of the forward propagating wave shifts into the Doppler shifted continuum of the backward propagating wave.

\section{Waves in a Non-uniform tube}

Now let us consider a tube with a smooth variation of density and flow across the loop cross-section. In particular we consider the case when $\rho$ varies from its internal value $\rho_{\mathrm{i}}$ to its external value $\rho_{\mathrm{e}}$ in the interval $[R-l / 2, R+l / 2]$ and the velocity changes from $v_{\mathrm{i}}$ to 0 in the interval $\left[R-l^{\star} / 2, R+l^{\star} / 2\right]$. Under such conditions the process of resonant absorption takes place and kink oscillations in coronal loops will damp efficiently. The reader is referred to Goossens (2008) and references therein for a detail review on this kink wave damping mechanism.

As in the previous Section we concentrate on propagating waves, the possible excitation of standing waves in the presence of flow is discussed later.

\subsection{The TTTB approximation}

In a non-uniform tube the imaginary part of the frequency (of the trapped propagating modes) is different from zero due to mode conversion at the inhomogeneous layer $\left(\omega=\omega_{R}+i \gamma\right)$. Some time ago Goossens et al. (1992) derived an expression for the damping rate in the thin tube and thin boundary $(l \ll R)$ approximation for incompressible MHD waves. For compressible waves in a magnetic cylinder, using the loop model considered here, we obtain exactly the same expression, given by (see their Eq. (76))

$$
\gamma=-\frac{\rho_{\mathrm{i}}^{2}\left(\Omega_{\mathrm{i}}^{2}-\omega_{\mathrm{A}, \mathrm{i}}^{2}\right)^{2}}{2\left(\rho_{\mathrm{i}}+\rho_{\mathrm{e}}\right) \omega_{\mathrm{cm}}} \frac{1}{\rho\left(r_{\mathrm{A}}\right)|\Delta|} \frac{|m| \pi}{r_{\mathrm{A}}} .
$$

As usual $r_{\mathrm{A}}$ is the resonant position that is calculated from

$\Omega^{2}\left(r_{\mathrm{A}}\right)=\omega_{\mathrm{A}}^{2}\left(r_{\mathrm{A}}\right)$,

i.e. where there is a match between the Doppler shifted frequency and the local Alfvén frequency. It is assumed that the real part of the frequency of the resonantly damped mode is given by Eq. (1). From a physical point of view, the condition given by Eq. (9) means that the eigenmodes resonantly interact with the Alfvén continuum, which is Doppler shifted as a result of flow.

The factor $\Delta$ in the denominator of Eq. (8) is

$\Delta=\frac{\mathrm{d}}{\mathrm{d} r}\left(\Omega^{2}(r)-\omega_{\mathrm{A}}^{2}(r)\right)$,

which contains a term with the derivative of the flow in the radial direction, absent in the static situation, that can increase or decrease the value of $\Delta$.

Given a particular density and velocity profile, the different variables in Eq. (8) can be evaluated. For simplicity, we use the well known sinusoidal profile for the density given by

$\rho(r)= \begin{cases}\rho_{\mathrm{i}}, & 0 \leq r<R-l / 2 \\ \frac{\rho_{\mathrm{i}}}{2}\left[\left(1+\frac{\rho_{\mathrm{e}}}{\rho_{\mathrm{i}}}\right)-\left(1-\frac{\rho_{\mathrm{e}}}{\rho_{\mathrm{i}}}\right) \sin \frac{\pi(r-R)}{l}\right], & R-l / 2 \leq r \leq R+l / 2 \\ \rho_{\mathrm{e}}, & r>R+l / 2\end{cases}$

This convenient profile has been used in many studies about resonant absorption (e.g. Ruderman \& Roberts 2002; Van Doorsselaere et al. 2004; Arregui et al. 2005; Terradas et al. 2006). In order to make the mathematical approach more tractable we also assume that the variation of the flow speed is sinusoidal inside the loop layer, i.e.,

$v(r)= \begin{cases}v_{\mathrm{i}}, & 0 \leq r<R-l^{\star} / 2, \\ \frac{v_{i}}{2}\left[1-\sin \frac{\pi(r-R)}{l^{\star}}\right], & R-l^{\star} / 2 \leq r \leq R+l^{\star} / 2, \\ 0, & r>R+l^{\star} / 2 .\end{cases}$

The flow is variable over a layer of thickness $l^{\star}$ which is not necessarily equal to the characteristic thickness of the layer $l$ where the density is non-constant. In general, in preceding studies it 


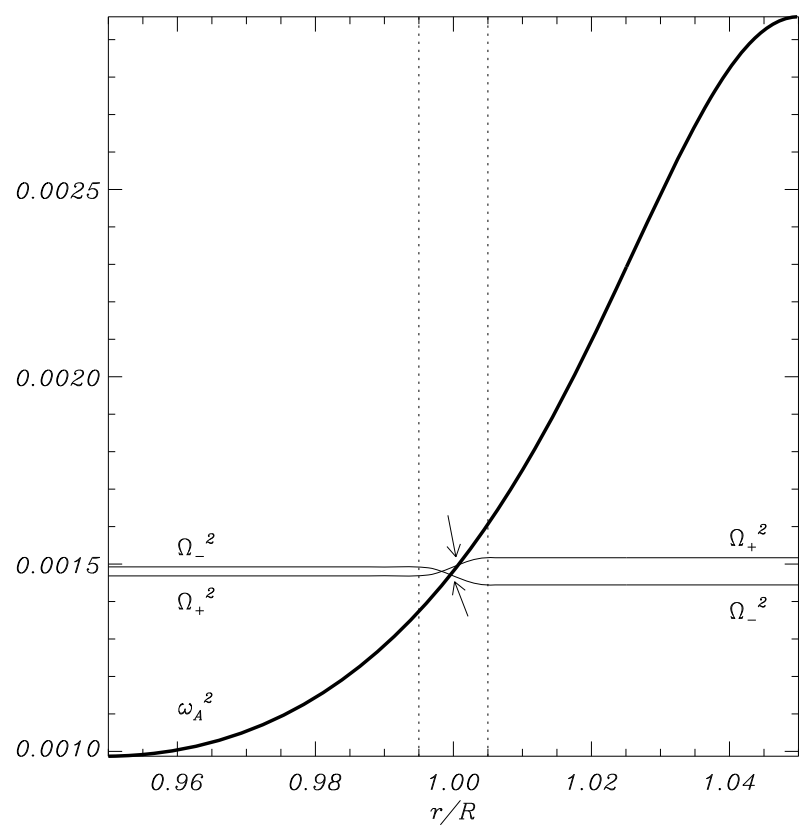

Fig. 2. Forward $\left(\Omega_{+}\right)$, backward $\left(\Omega_{-}\right)$and local Alfvén frequency (thick line) as a function of the radial position. The arrows indicate the location of the resonances. In this plot $v_{\mathrm{i}} / v_{\mathrm{Ai}}=0.02, l / R=0.1, l^{\star} / R=0.01$, $L=100 R$ and $\rho_{\mathrm{i}} / \rho_{\mathrm{e}}=3$. The dotted vertical lines mark the limits of the inhomogeneity of the flow.

has been assumed a discontinuous flow (at the boundary of the loop), except by Erdélyi \& Taroyan (2003a,b) who considered a linear profile to model MHD waves in the Earth's magnetotail.

For the profiles given by Eqs. (11) and (12) it turns out that Eq. (9) is a transcendental equation for the resonant position $r_{\mathrm{A}}$. This equation is solved using standard numerical techniques. Depending on the spatial scales of the density and velocity we distinguish two different regimes, $l^{\star} \gtrsim l$ and the asymptotic case $l^{\star} \ll l$.

The analysis of the first situation is rather simple (see also Peredo \& Tataronis 1990), since the the forward propagating wave has always a single resonant position in the range $R<$ $r_{\mathrm{A}}<R+l / 2$, while the resonant position of the backward wave is situated in the range $R-l / 2<r_{\mathrm{A}}<R$. This behaviour is easily understood from Fig. 2, where we have plotted the Doppler shifted frequencies and the Alfvén frequency as a function of the radial coordinate. The resonant positions are located at the intersection of $\Omega^{2}$ with $\omega_{\mathrm{A}}^{2}$ (see arrows). Note that Fig. 2 also shows that if the Alfvén frequency is discontinuous (jump in density, $l=0$ ) there are no resonances (implying no damping) since $\Omega^{2}$ will never intersect the curve corresponding to $\omega_{\mathrm{A}}^{2}$.

Once the resonant position $r_{\mathrm{A}}$ is determined $|\Delta|_{r_{\mathrm{A}}}$ is evaluated and we finally obtain the value of the damping rate $\gamma$ (using Eq. (8)). A useful quantity that we can calculate is the the damping per period, given by

$\frac{\tau_{\mathrm{D}}}{P}=\frac{\left|\omega_{\mathrm{kf}}\right|}{|\gamma|} \frac{1}{2 \pi}$.

In this expression we use the real part of the frequency given by Eq. (1). In Fig. 3 (see solid lines) $\tau_{\mathrm{D}} / P$ is represented for two different values of the characteristic widths of the layers, $l / R=l^{\star} / R=0.05,0.1$, as a function of the internal flow (recall that both the frequency and the damping rate depend on the flow). The curves with positive slope correspond to the forward waves while the ones with the negative slope represent the backward wave. Figure 3 also shows that increasing the strength of

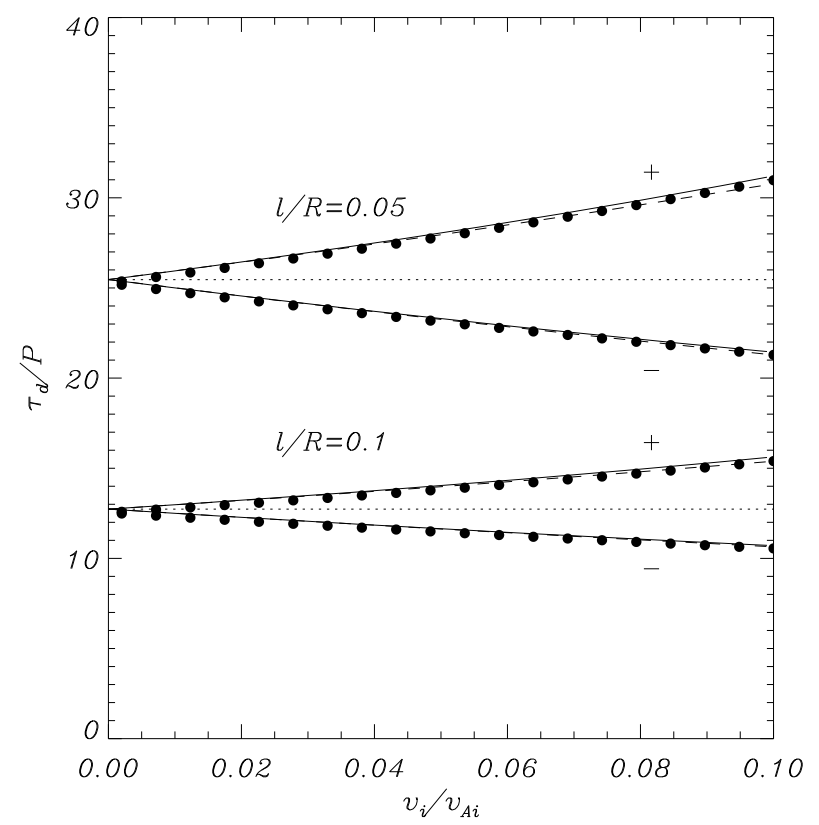

Fig. 3. Damping per period as a function of the flow inside the loop for the forward (+) and backward (-) propagating waves. The solid lines represent the analytical results calculated using Eqs. (1) and (8). The dashed lines are the approximations of the damping per period using Eqs. (17) and (28). The dots represent the full numerical solution of the resistive eigenvalue problem. The horizontal dotted lines are the damping per period in the static situation. For the curves with $l / R=0.05,0.1$ we have used $l^{\star} / R=0.05,0.1$.

the flow increases the damping per period for the forward wave and decreases it for the backward wave. This is in agreement with the results of Peredo \& Tataronis (1990), the shifts of the frequency modify the location of the resonant surfaces in such a way that one of the natural modes is closer to the resonance while the other is further away from the resonance relative to the static situation. As a consequence, one mode is damped more efficiently than the other. Nevertheless, the effect of the flow does not significantly change the damping per period for the regime considered here $\left(0<v_{\mathrm{i}} / v_{\mathrm{Ai}}<0.1\right)$. Hence the mechanism of resonant absorption is very robust in the presence of an internal flow. The thicker the layer, the faster the attenuation (cf., the results for $l / R=0.1$ with the results for $l / R=0.05$ ), a result already known for the static case. We also see that the change of $\tau_{\mathrm{D}} / P$ is quite smooth with respect to $v_{\mathrm{i}}$.

In Fig. 4 we have represented the damping per period as a function of $l^{\star}$ in units of loop radius for two different values of $l / R$. In this plot, $l$, the characteristic scale of the density transition, is fixed (recall we are still in the regime with $l^{\star} \gtrsim l$ ). For large values of $l^{\star}$ compared to $l$ we see that the dependence is quite weak with the thickness of the flow profile. The forward propagating wave has a larger damping per period than the backward propagating wave. However, when $l^{\star} \lesssim l$ the situation is reversed. The curves cross and the forward propagating wave is attenuated faster than the backward propagating wave, indicating that we are at the threshold of a different regime.

Now let us concentrate on the situation when $l^{\star} \ll l$, i.e., we investigate the case with a very steep profile for the equilibrium flow velocity. This is different to the regime discussed earlier $\left(l^{\star} \gtrsim l\right)$ in several aspects. In Fig. 5 we have plotted a typical example. It is easy to see that the forward wave can have now three different resonant positions (see arrows). Apart from the resonance inside the inhomogeneous velocity 
J. Terradas et al.: Resonantly damped kink oscillations with flow

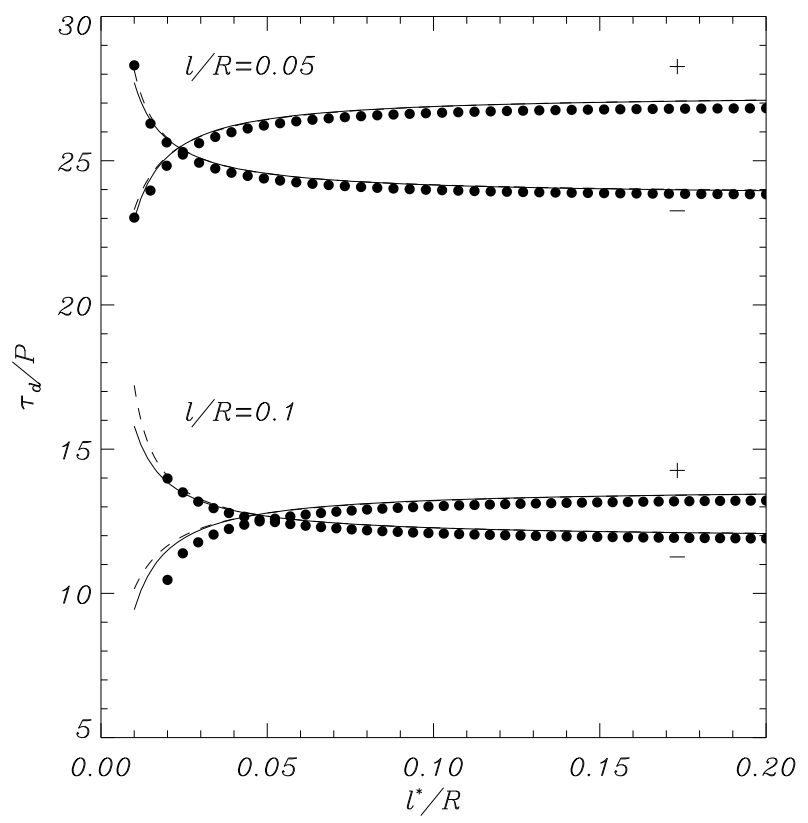

Fig. 4. Damping per period for the forward and backward propagating waves as a function of the width of the flow profile, $l^{\star}$. We use the same notation as in Fig. 3. In this plot $v_{\mathrm{i}} / v_{\mathrm{Ai}}=0.02$.

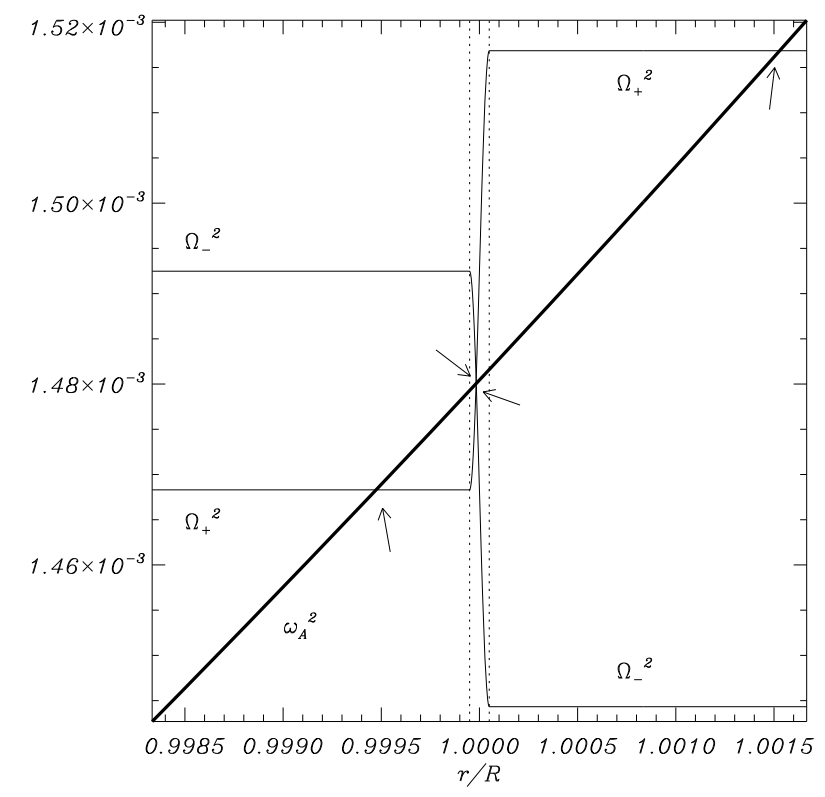

Fig. 5. Forward $\left(\Omega_{+}\right)$, backward $\left(\Omega_{-}\right)$and local Alfvén frequency (see thick line) as a function of the radial position. The arrows indicate the location of the resonances. In this plot $v_{\mathrm{i}} / v_{\mathrm{Ai}}=0.02, l / R=0.1$ and $l^{\star} / R=0.0001, L=100 R$ and $\rho_{\mathrm{i}} / \rho_{\mathrm{e}}=3$. The vertical dotted lines mark the limits of the inhomogeneity of the flow.

layer, the forward wave has two additional resonances, one at $R-l / 2<r_{\mathrm{A}}<R-l^{\star} / 2$ and the second at $R+l^{\star} / 2<r_{\mathrm{A}}<R+l / 2$. The backward wave still has a single resonance situated, as before, inside the velocity layer. Although the situation is more complicated, we can still understand the role of the resonances. The derivatives of $\Omega^{2}$ with respect to $r$ at the resonance inside the velocity layer, located around $r=R$ (for both the forward and backward waves), become very large (in absolute value) for small $l^{\star}$, thus dominating over the derivative of $\omega_{\mathrm{A}}^{2}$ (see Eq. (10)). This means that the factor $|\Delta|_{r_{A}}$ is large and tending to infinity (for $l^{\star} \ll l$ ), therefore $\gamma$ will tend to zero, i.e., they will not produce any damping. However, the other two resonances of the forward wave still behave as ordinary resonances since the derivative of the flow is zero (they are located outside the velocity layer where the flow is constant) and in this situation the total damping of the mode will be finite due to the combined contribution of the two resonances.

\subsection{Linear approximation of frequency and damping time in the TTTB approximation}

Visual inspection of Fig. 3 shows that the damping per period varies smoothly and appears, to very good approximation, to be a linear function of $v_{\mathrm{i}} / v_{\mathrm{A}, \mathrm{i}}$. This has motivated us to derive a linear approximation of the frequency $\omega_{\mathrm{kf}}$ and the damping rate $\gamma$ as function of $v_{\mathrm{i}} / v_{\mathrm{A}, \mathrm{i}}$. Actually, it turns out that

$x=\frac{k v_{\mathrm{i}}}{\omega_{\mathrm{k}}}$,

is a more convenient variable for obtaining the linear approximation. Since

$x=\frac{1}{\sqrt{2}}\left(\frac{\rho_{\mathrm{i}}+\rho_{\mathrm{e}}}{\rho_{\mathrm{i}}}\right)^{1 / 2} \frac{v_{\mathrm{i}}}{v_{\mathrm{A}, \mathrm{i}}}$,

it follows that $v_{\mathrm{i}} / v_{\mathrm{A}, \mathrm{i}} \ll 1$ is equivalent to $x \ll 1$.

The first order approximation to $\omega_{\mathrm{cm}}$ (given by Eq. (2)) is

$\omega_{\mathrm{cm}}=\omega_{\mathrm{k}}$,

so that the linear approximation to $\omega_{\mathrm{k}+}$ (for the forward wave) is

$\omega_{\mathrm{kf}+}=\omega_{\mathrm{k}}\left(1+x \frac{\rho_{\mathrm{i}}}{\rho_{\mathrm{i}}+\rho_{\mathrm{e}}}\right)$.

In order to derive a linear approximation to $\gamma$ we need some intermediate results to be used in Eq. (8). The linear approximation to quantity $\rho_{\mathrm{i}}\left(\Omega_{\mathrm{i}}^{2}-\omega_{\mathrm{A}, \mathrm{i}}^{2}\right)^{2}$ is

$\rho_{\mathrm{i}}\left(\Omega_{\mathrm{i}}^{2}-\omega_{\mathrm{A}, \mathrm{i}}^{2}\right)^{2}=\frac{\rho_{\mathrm{i}}^{2} \rho_{\mathrm{e}}^{2}}{\left(\rho_{\mathrm{i}}+\rho_{\mathrm{e}}\right)^{2}}\left(\omega_{\mathrm{A}, \mathrm{e}}^{2}-\omega_{\mathrm{A}, \mathrm{i}}^{2}\right)^{2}\left\{1-4 \frac{\omega_{\mathrm{k}}^{2}}{\omega_{\mathrm{A}, \mathrm{e}}^{2}-\omega_{\mathrm{A}, \mathrm{i}}^{2}} x\right\}$,

and the linear approximation to $\rho\left(r_{\mathrm{A}}\right) \Delta$ can be written as

$\rho\left(r_{\mathrm{A}}\right) \Delta=\rho\left(r_{\mathrm{A}}\right) \omega_{\mathrm{k}}^{2}\left\{\frac{x}{l_{v}}-\frac{(1+y)}{l_{\rho}}\right\}$.

Here $l_{\rho}$ and $l_{v}$ are the length scales of variation of density $\rho$ and velocity $v_{i}$, respectively. They are defined as

$\frac{1}{l_{\rho}}=\frac{1}{\rho\left(r_{\mathrm{A}}\right)}\left|\frac{\mathrm{d} \rho}{\mathrm{d} r}\right|_{r_{\mathrm{A}}}$

$\frac{1}{l_{\mathrm{v}}}=\frac{1}{v\left(r_{\mathrm{A}}\right)}\left|\frac{\mathrm{d} v}{\mathrm{~d} r}\right|_{r_{\mathrm{A}}}$

Note that $l_{\rho}$ and $l_{\mathrm{v}}$ are not equal to the width of the non-uniform layer of density $l$ nor to the width of the non-uniform layer of velocity $l^{\star}$. E.g, for the sinusoidal profile $v\left(r_{\mathrm{A}}\right)=v_{\mathrm{i}} / 2$ (since we assume that $\left.r_{\mathrm{A}}=R\right)$ and $\rho\left(r_{\mathrm{A}}\right)=\left(\rho_{\mathrm{i}}+\rho_{\mathrm{e}}\right) / 2$, the resultant characteristic spatial scales are

$l_{\rho}=\frac{l}{\pi} \frac{\rho_{\mathrm{i}}+\rho_{\mathrm{e}}}{\rho_{\mathrm{i}}-\rho_{\mathrm{e}}}, \quad l_{v}=\frac{l^{\star}}{\pi}$.

The quantity $y$ in Eq. (19) is defined as

$y=x \frac{\rho_{\mathrm{i}}-\rho_{\mathrm{e}}}{\rho_{\mathrm{i}}+\rho_{\mathrm{e}}}$. 
If we assume that $l_{\rho} \approx l_{v}$ then

$$
\frac{x}{l_{v}} \ll \frac{(1+y)}{l_{\rho}} .
$$

In this case the linear approximation to $1 /\left(\rho\left(r_{\mathrm{A}}\right)|\Delta|_{r_{\mathrm{A}}}\right)$ is

$$
\frac{1}{\rho\left(r_{\mathrm{A}}\right)|\Delta|_{r_{\mathrm{A}}}}=\frac{l_{\rho}}{\rho\left(r_{\mathrm{A}}\right) \omega_{\mathrm{k}}^{2}}(1-y+x J) \text {. }
$$

Here $J$ is a factor which measures the relative importance of the non-uniformity of the flow to that of density and it is defined as

$$
J=\frac{l_{\rho}}{l_{v}} .
$$

With all terms approximated linearly, the approximation of the damping rate, $\gamma$, becomes

$$
\begin{aligned}
\gamma= & -\frac{\pi}{8} \frac{l_{\rho}}{\rho\left(r_{\mathrm{A}}\right)} \frac{\left(\rho_{\mathrm{i}}-\rho_{\mathrm{e}}\right)^{2}}{\rho_{\mathrm{i}}-\rho_{\mathrm{e}}} \omega_{\mathrm{k}} \\
& \times\left\{1+x\left[-\frac{8 \rho_{\mathrm{i}} \rho_{\mathrm{e}}}{\left(\rho_{\mathrm{i}}-\rho_{\mathrm{e}}\right)\left(\rho_{\mathrm{i}}+\rho_{\mathrm{e}}\right)}-\frac{2 \rho_{\mathrm{i}}}{\rho_{\mathrm{i}}+\rho_{\mathrm{e}}}+\frac{2 v\left(r_{\mathrm{A}}\right)}{v_{\mathrm{i}}}+J\right]\right\} .
\end{aligned}
$$

If we repeat the analysis for the backward wave we obtain the same expression with a change in the sign in front of $x$.

The final expression for the damping rates of the two waves (forward and backward propagating) using the sinusoidal profile of density and velocity reduces to

$$
\begin{aligned}
\gamma= & -\frac{1}{4} \frac{l}{R} \frac{\left(\rho_{\mathrm{i}}-\rho_{\mathrm{e}}\right)}{\left(\rho_{\mathrm{i}}+\rho_{\mathrm{e}}\right)} \omega_{\mathrm{k}} \\
& \times\left\{1 \pm \frac{k v_{\mathrm{i}}}{\omega_{\mathrm{k}}}\left[-\frac{8 \rho_{\mathrm{i}} \rho_{\mathrm{e}}}{\left(\rho_{\mathrm{i}}-\rho_{\mathrm{e}}\right)\left(\rho_{\mathrm{i}}+\rho_{\mathrm{e}}\right)}-\frac{\rho_{\mathrm{i}}-\rho_{\mathrm{e}}}{\rho_{\mathrm{i}}+\rho_{\mathrm{e}}}+\frac{\left(\rho_{\mathrm{i}}+\rho_{\mathrm{e}}\right)}{\left(\rho_{\mathrm{i}}-\rho_{\mathrm{e}}\right)} \frac{l}{l^{\star}}\right]\right\} .
\end{aligned}
$$

The \pm sign corresponds to forward and backward propagating waves respectively. When the flow is zero, the second term inside the curly brackets vanishes and we recover the formula of the damping in the static equilibrium (see for example Ruderman $\&$ Roberts 2002). In the non-static case since we have assumed that $l_{\rho} \approx l_{v}\left(l \approx l^{\star}\right)$ the last two terms inside the square brackets are of the same order and we see that the imaginary part of the frequency linearly decreases with the flow for the forward propagating wave and increases for the backward wave. Using the linear approximation to the frequency (Eq. (17)) and damping rate (Eq. (28)) it is straight forward to calculate the damping per period. The results are represented in Fig. 3 (see dashed lines). We see that the analytical approximations agree very well with the full solution based on the calculation of $r_{\mathrm{A}}$ and the evaluation of Eq. (1) and Eq. (8). Note that the dependence of the damping rate on the spatial variation of the flow across the loop boundary, $l^{\star}$, is present in Eq. (28). In Fig. 4 we have plotted the results using this expression (see dashed lines). Again, we find an excellent agreement between the two curves for both the forward and backward waves.

For the regime $l^{\star} \ll l$ it is possible to derive useful information from the linear approximation. Using the assumption $l_{v} \ll l_{\rho}$ it is easy to see that the damping rate of the resonance inside the velocity layer (for both the forward and backward waves) is proportional to $l^{\star}$, which means, as we have already anticipated in Sect. 3.1, that the contribution of this resonance to the total damping tends to zero (damping time tending to infinity) for a purely discontinuous velocity profile. This is the behaviour already found in Fig. 4 for the backward wave. Moreover, we can estimate the total damping of the two regular resonances of the forward wave (see Fig. 5) by adding the individual damping rates. It turns out that the total damping rate for the forward wave is simply

$$
\begin{aligned}
\gamma= & -\frac{1}{2} \frac{l}{R} \frac{\left(\rho_{\mathrm{i}}-\rho_{\mathrm{e}}\right)}{\left(\rho_{\mathrm{i}}+\rho_{\mathrm{e}}\right)} \omega_{\mathrm{k}} \\
& \times\left\{1-\frac{k v_{\mathrm{i}}}{\omega_{\mathrm{k}}}\left[\frac{8 \rho_{\mathrm{i}} \rho_{\mathrm{e}}}{\left(\rho_{\mathrm{i}}-\rho_{\mathrm{e}}\right)\left(\rho_{\mathrm{i}}+\rho_{\mathrm{e}}\right)}+\frac{\rho_{\mathrm{i}}-\rho_{\mathrm{e}}}{\rho_{\mathrm{i}}+\rho_{\mathrm{e}}}\right]\right\} .
\end{aligned}
$$

This is the asymptotic value for the forward wave when $l^{\star}$ tends to zero. Note that this is twice the damping rate of the situation with a very smooth velocity profile (see Eq. (28) when $l^{\star} \gg l$ ) indicating a more efficient attenuation (half the damping time).

\subsection{Beyond the TTTB approximation: full resistive eigenvalue problem}

The results of the previous Sections are based on the TT approximation. It is known that without flows this approximation works very well even for thick layers. However it remains to be confirmed whether this assumption is still valid in the presence of flows. For this reason, we go beyond the TTTB approximation. In this case we solve the full problem numerically. We follow the approach of Terradas et al. (2006). To study the quasimode properties, the eigenvalue problem given by Eqs. (1)-(5) in Terradas et al. (2006), plus the additional terms due to the flow, is solved. A time dependence of the form $\mathrm{e}^{\mathrm{i} \omega t}$ is assumed and the problem is solved numerically using a code based on finite elements. As boundary conditions we impose that the velocity components are zero for $r \rightarrow \infty$. In practice, the condition is applied at $r=r_{\max }$, and then it is necessary to check that the results do not depend on this parameter. On the other hand, at $r=0$ it is imposed that $\partial v_{r} / \partial r=0$, i.e., we select the regular solution at the origin, while the rest of the variables are extrapolated. All the variables and the eigenfrequency are assumed to be complex numbers, since we are interested in resonantly damped modes. We include resistivity to avoid the singular behaviour of the ideal MHD equations at the resonances. The resistive eigenvalue problem is solved and we obtain the real and the imaginary part of the frequency which must be independent of the magnetic Reynolds number that we use in the computations (Poedts \& Kerner 1991).

The results of the calculations for $l^{\star} \gtrsim l$ are plotted in Fig. 3 (shown by dots). The agreement with the analytical calculations, using the TTTB approximation, is remarkable. The numerical curves almost overlap with the analytical ones. In Fig. 4 (shown by dots) we represent the damping per period as a function of $l^{\star}$ and find the same behaviour as in the analytical expression. With these results we are even more confident about the method used in Sect. 3.1 and about the analytical expressions derived in Sect. 3.2.

For the regime $l^{\star} \ll l$ the numerical method we are using fails since the thinner the layer (in density or velocity) the larger the Reynolds number required for the damping time to be independent of the dissipation. A method based on the application of the jump conditions at the resonance or resonances, used for example by Tirry et al. (1998) or Andries et al. (2000); Andries \& Goossens (2001), is more appropriate but since this is not the main focus of this paper it will not be further investigated here.

\subsection{The standing wave problem}

The results presented in the previous sections correspond to two propagating waves, one propagating in the direction of the flow, $\omega_{\mathrm{f}+}$, and the other travelling in the opposite direction, $\omega_{\mathrm{f}_{-}}$. In 
general, an initial perturbation will excite these two modes at the same time and the system will oscillate with a combination of the two frequencies. If the frequencies are real the superposition of the two propagating modes (with the same amplitude and phase) will have the following form (recall that $\omega_{\mathrm{f}-}$ is negative for slow flows)

$$
\begin{aligned}
\cos \left(\omega_{\mathrm{f}+} t-k z\right)+ & \cos \left(\left|\omega_{\mathrm{f}-}\right| t+k z\right)= \\
& \cos (k z)\left[\cos \left(\omega_{\mathrm{f}+} t\right)+\cos \left(\left|\omega_{\mathrm{f}-}\right| t\right)\right] \\
+ & \sin (k z)\left[\sin \left(\omega_{\mathrm{f}+} t\right)-\sin \left(\left|\omega_{\mathrm{f}-}\right| t\right)\right] .
\end{aligned}
$$

Strictly speaking to have a standing wave it is required that $\omega_{\mathrm{f}_{+}}=$ $\left|\omega_{\mathrm{f}-}\right|$. This is true only in the absence of flow. Mathematically, it is impossible to form a standing wave in the presence of longitudinal flow. However, for slow flows (the regime suggested by the observations), the frequencies of the forward and backward waves hardly differ and a quasi-standing pattern may be formed. Under such conditions the dominant temporal part in Eq. (30) is

$$
\begin{aligned}
& \cos \left(\omega_{\mathrm{f}+} t\right)+\cos \left(\left|\omega_{\mathrm{f}-}\right| t\right)= \\
& 2 \cos \left(\frac{\omega_{\mathrm{f}+}+\left|\omega_{\mathrm{f}-}\right|}{2} t\right) \cos \left(\frac{\omega_{\mathrm{f}+}-\left|\omega_{\mathrm{f}-}\right|}{2} t\right) .
\end{aligned}
$$

This equation shows that the loop has an oscillation frequency given by $\frac{\omega_{\mathrm{f}+}+\left|\omega_{\mathrm{f}-}\right|}{2}$ modulated by an envelope that oscillates at the beating frequency $\frac{\omega_{f_{+}-}\left|\omega_{\mathrm{f}}\right|}{2}$. The beating frequency is very low (i.e., a very large envelope), meaning that to all practical purposes the behaviour of the system is equivalent to that of a standing wave.

It must be noted that fact that the amplitude of the oscillation is quickly damped with time, as the observations indicate, might favour the formation of a standing wave when the flow is present. In this situation, when the damping times of the forward and backward waves are shorter than the beating period $\left(|\gamma| \gg \frac{\left.\omega_{\mathrm{f}_{+}-\left|\omega_{\mathrm{f}-}\right|}\right)}{2}\right.$ the envelope of the signal is dominated by the attenuation due to resonant absorption rather than by the modulation due to the beating.

\section{Conclusions and discussion}

We have studied the effect of a longitudinal flow on propagating kink oscillations of a coronal loop and their damping, and have shown, in agreement with previous studies, that under typical coronal conditions a longitudinal flow, which is highly subAlfvénic, is unable to produce $\mathrm{KH}$-unstable modes. It was also demonstrated that leaky modes are generated by fast flows that have velocities comparable to the local Alfvén velocity. Since observations show that flows are at most, $10 \%$ of the Alfvén speed, this means that forward and the backward waves must always be trapped in coronal loops. Moreover, the forward wave never enters into the Doppler shifted continuum of the backward propagating waves (see Fig. 1a) and so there are no resonant flow instabilities for slow flows. Although instabilities due to longitudinal flows are unlikely to occur in coronal loops, other kinds of instabilities, for example produced by the azimuthal shear of the kink mode are possible (see Terradas et al. 2008a; Terradas 2009; Clack \& Ballai 2009; Soler et al. 2010; Zaqarashvili et al. 2010).

It was demonstrated that the resonant damping mechanism due to non-uniform density and flow at the loop boundary is not significantly altered by the presence of the flow as long as the scale of inhomogeneity of the flow is similar or larger than the scale of inhomogeneity of the density. We derived simple expressions for the linear approximation to the frequency and damping rate as a function of the flow, for forward and backward propagating waves in the TTTB limit. These simple formulae are very accurate, since they agree very well with the numerical calculations of the full resistive eigenvalue problem. The analytical expressions will facilitate future seismological applications (along the lines of those proposed by Arregui et al. 2007; Goossens et al. 2008), since now the damping rate contains the velocity flow as an additional parameter. Using these expressions we can estimate the differences with respect to the static situation. For example, for a loop with flows of $v_{\mathrm{i}}=0.1 v_{\mathrm{Ai}}$ and a thickness of the layer in density and velocity of $0.05 R$, the period decreases a $6 \%$ and the damping time increases a $14 \%$ for the forward wave, while for the backward wave the period increases a $6 \%$ and the damping time decreases a $11 \%$ compared to the purely static equilibrium case.

A physically peculiar situation takes place when the flow has a sharp transition at the loop boundary (in the limit of $l^{\star} \ll l$ ). The backward wave is transformed into an undamped mode even in the presence of a non-uniform density transition. Conversely, the forward wave is more efficiently damped due to the introduction of two new resonances outside the velocity transition layer.

Finally, we must point out that the problem studied in this paper is an initial value problem where the wavenumber, $k$, is assumed to be real, and we solve for the complex frequency $\omega$. Nevertheless, a more convenient description of certain coronal loop problems would require to study the boundary value problem, where the frequency is prescribed and one solves for the complex longitudinal wavenumber. This is will the subject of a future work.

Acknowledgements. J.T. and M.G. acknowledge support from K.U. Leuven via GOA/2009-009. J.T. acknowledges the funding provided under projects AYA2006-07637 (Spanish Ministerio de Educación y Ciencia) and PCTIB2005GC3-03 (Conselleria d'Economia, Hisenda i Innovació of the Government of the Balearic Islands). In addition, J.T. thanks Jesse Andries, Gary Verth and Roberto Soler for their useful suggestions that helped to improve the original manuscript. The present research was initiated while I.B. was a guest at Dept. of Physics, UIB (Spain). I.B. acknowledges the financial support and warm hospitality of the Dept. of Physics, UIB. I.B. was supported by NFS Hungary (OTKA, K67746) and The National University Research Council Romania (CNCSIS-PN-II/531/2007). We are grateful as well to an anonymous referee whose comments and suggestions helped us to improve the paper.

\section{References}

Andries, J., \& Goossens, M. 2001, A\&A, 368, 1083

Andries, J., Tirry, W. J., \& Goossens, M. 2000, ApJ, 531, 561

Arregui, I., Van Doorsselaere, T., Andries, J., Goossens, M., \& Kimpe, D. 2005, A\&A, 441, 361

Arregui, I., Andries, J., Van Doorsselaere, T., Goossens, M., \& Poedts, S. 2007, A\&A, 463, 333

Aschwanden, M. J., Fletcher, L., Schrijver, C. J., \& Alexander, D. 1999, ApJ, 520,880

Brekke, P., Kjeldseth-Moe, O., \& Harrison, R. A. 1997, Sol. Phys., 175, 511

Cally, P. S. 1986, Sol. Phys., 103, 277

Cally, P. S. 2003, Sol. Phys., 217, 95

Chae, J., Ahn, K., Lim, E.-K., Choe, G. S., \& Sakurai, T. 2008, ApJ, 689, L73

Chandrasekhar, S. 1961, Hydrodynamic and hydromagnetic stability, ed. S. Chandrasekhar

Clack, C. T. M., \& Ballai, I. 2009, Phys. Plasmas, 16, 072115

Erdélyi, R., \& Goossens, M. 1996, A\&A, 313, 664

Erdélyi, R., Goossens, M., \& Ruderman, M. S. 1995, Sol. Phys., 161, 123

Erdélyi, R., \& Taroyan, Y. 2003a, in Solar Wind Ten, ed. M. Velli, R. Bruno, F. Malara, \& B. Bucci, AIP Conf. Ser., 679, 355

Erdélyi, R., \& Taroyan, Y. 2003b, J. Geophys. Res. (Space Physics), 108, 1043

Ferrari, A., Trussoni, E., \& Zaninetti, L. 1981, MNRAS, 196, 1051

Goossens, M. 2008, in IAU Symp., 247, 228 
Goossens, M., Arregui, I., Ballester, J. L., \& Wang, T. J. 2008, A\&A, 484, 851 Goossens, M., Hollweg, J. V., \& Sakurai, T. 1992, Sol. Phys., 138, 233 Goossens, M., Terradas, J., Andries, J., Arregui, I., \& Ballester, J. L. 2009, A\&A, 503,213

Hollweg, J. V., Yang, G., Cadez, V. M., \& Gakovic, B. 1990, ApJ, 349, 335

Nakariakov, V. M., Ofman, L., Deluca, E. E., Roberts, B., \& Davila, J. M. 1999, Science, 285,862

Ofman, L., \& Wang, T. J. 2008, A\&A, 482, L9

Peredo, M., \& Tataronis, J. A. 1990, Washington DC American Geophysical Union Geophysical Monograph Series, 58, 289

Poedts, S., \& Kerner, W. 1991, Phys. Rev. Lett., 66, 2871

Ruderman, M. S., \& Goossens, M. 1995, J. Plasma Phys., 54, 149

Ruderman, M. S., \& Roberts, B. 2002, ApJ, 577, 475

Soler, R., Oliver, R., \& Ballester, J. L. 2008, ApJ, 684, 725

Soler, R., Terradas, J., Oliver, R., Ballester, J. L., \& Goossens, M. 2010, ApJ, 712,875
Terra-Homem, M., Erdélyi, R., \& Ballai, I. 2003, Sol. Phys., 217, 199 Terradas, J. 2009, Space Sci. Rev., 149, 255

Terradas, J., Oliver, R., \& Ballester, J. L. 2006, ApJ, 642, 533

Terradas, J., Andries, J., Goossens, M., et al. 2008a, ApJ, 687, L115

Terradas, J., Arregui, I., Oliver, R., \& Ballester, J. L. 2008b, ApJ, 678, L153

Tirry, W. J., Cadez, V. M., Erdélyi, R., \& Goossens, M. 1998, A\&A, 332, 786

Van Doorsselaere, T., Andries, J., Poedts, S., \& Goossens, M. 2004, ApJ, 606, 1223

Vasheghani Farahani, S., Van Doorsselaere, T., Verwichte, E., \& Nakariakov, V. M. 2009, A\&A, 498, L29

Winebarger, A. R., DeLuca, E. E., \& Golub, L. 2001, ApJ, 553, L81

Winebarger, A. R., Warren, H., van Ballegooijen, A., DeLuca, E. E., \& Golub, L. 2002, ApJ, 567, L89

Zaqarashvili, T. V., Díaz, A. J., Oliver, R., \& Ballester, J. L. 2010, A\&A, in press 